\title{
Parasites and microbiota: A complex relationship
}

\author{
Editorial Hala S Elwakil
}

Medical Parasitology Department, Faculty of Medicine, Ain Shams University, Cairo, Egypt

The human gut is populated by a huge number of microbiota that is acquired first at birth and soon stabilizes in the early years of life. Microflora and gut helminths have co-evolved over many millions of years until recently when the geographical prevalence of parasitic diseases in human has become restricted to the developing world. Immune homeostasis relies on a balance in the composition of intestinal microflora; long lived parasites have also been shown to regulate immune function, and their absence in western lifestyles is suggested to be a factor for the increasing frequency of allergy and autoimmunity. The diversity of the microbiota is thought to reflect the health of the intestine and contributes to healthy metabolic and immune functioning.

Keywords: gut helminthes, immune homeostasis, intestinal microflora, microbiota.

Received: 12 November, 2018, Accepted: 24 November, 2018

Corresponding Author: Hala S Elwakil, Tel. : 01001241275, E-mail: halawakil39@gmail.com

Print ISSN: 1687-7942, Online ISSN: 2090-2646, Vol. 11, No. 3, December, 2018.

The intestinal environment can be considered as an ecosystem where biological and chemical interactions occur at various organizational levels between host, parasites, and microbial communities. It has been suggested that the interdependence relationship between the host microbiota, host immune system and intestinal parasites shape the clinical outcome of enteric infections ${ }^{(1)}$.

Helminth infection modifies intestinal microflora: The changes in the composition of the gut microbiota that associate helminths infection may be due to the secretion of anti-microbial components by the parasite, the disruption of the epithelial barrier by the parasite that alters the intestinal environment or the stimulation of specific immune responses ${ }^{(2)}$. Indeed, alterations in composition of bacterial communities were found in stool samples of school children with nematodes infection living in rural Ecuador ${ }^{(3)}$. This study found decreased abundance of Clostridia, with reduction in overall bacterial diversity in stool samples of children co-infected with T. trichiura and A. lumbricoides. In a comparative study between fecal stool samples from helminthinfected or non-infected individuals living in rural Malaysia, Lee et al., ${ }^{(4)}$ reported a significant increase in bacterial diversity among individuals infected with any helminth species, and an increased abundance of Paraprevotellaceae family in those individuals infected only with T. trichiura.

Modification in microflora alters the Th17/T regulatory cell balance: Changes in the symbiotic microbiota play a role in the balance between inflammatory Th17 cells and regulatory Foxp3 ${ }^{+}$ cells functioning in the intestine. The segmented filamentous bacteria were shown to be responsible for powerful Th17 responses and secretion of IL-
17 and IL-22, which enhances the resistance to the intestinal pathogen $C$. rodentium ${ }^{(5)}$. Conversely, Clostridium species are responsible for the induction of IL-10 plus Foxp3 ${ }^{+}$regulatory cells in the colon ${ }^{(6)}$. Bancroft et al., ${ }^{(1)}$ have shown that broad spectrum antibiotic treatment of mice infected with T. muris can significantly downregulate the levels of IL17 produced and alter the broader host immune response to T. muris.

Alterations in the TH17/Foxp3 balance maintains parasite survival: Helminths desire survival for extended periods to maximize egg output, thereby facilitating transmission. They trigger immune responses that include potent regulatory components, by the generation of induced $\mathrm{T}$ regs (iTregs), capable of controlling pathology induced by parasite invasion of the mucosa. D'Elia et al.,(7) suggested that Foxp3 $3^{+}$Tregs could protect the host from intestinal pathology associated with T. muris infection.

The effect of microbiota on the outcome of many parasitic infections: Reynold et al., ${ }^{(2)}$ suggested that the composition of microbiota may alter the priming of the murine immune system to alter susceptibility to helminth infection. It was found that while germ free mice are more susceptible to infections with bacterial or viral agents, persistence of the gastrointestinal helminth parasite Heligmosomoides polygyrus is markedly reduced in these animals compared with conventionally raised mice ${ }^{(8)}$. Those researchers found that $H$. polygyrus raises Lactobacillus taiwanensis abundance in the duodenum of C57BL/6 mice, which are highly susceptible to $H$. polygyrus infection, but not in BALB/c mice, which are relatively resistant. Moreover, experimental administration of $L$. taiwanensis to $\mathrm{BALB} / \mathrm{c}$ mice 
elevates regulatory $\mathrm{T}$ cell frequencies and results in greater helminth establishment. As regards the effects of the microbiota on host immune response to protozoal infections, when T. gondii reaches the gut of an individual with a functional microbiota, an immune response is initiated at the level of the intestinal mucosa by activation of toll-like receptors (TLR11/12) in dendritic cells. This recognition activates the transcription factor interferon (INF) regulatory factor 8 , leading to the production of interleukin 12 (IL-12) which promotes a cellularbased immunity with production of INF- $\gamma$ and differentiation of Th1 T lymphocytes. TLR-knockout mice (TLR11\%) are not completely impaired to respond to $T$. gondii as long as gut commensal bacteria are present because the production of diminished amounts of IL-12 is compensated by indirect stimulation provided by the gut microbial commensals via TLR2, TLR4, and TLR9. Therefore, TLR $11^{-}$mice survive the acute phase of infection. When TLR $11^{\%}$ mice are treated with antibiotics to eliminate gut commensal bacteria, this remaining protective IL-12 response is lost ${ }^{(9)}$. In addition, it was found that gut microbiota elicits a protective immune response against malaria transmission ${ }^{(10)}$. In a mouse study, Yelmiz et al., ${ }^{(10)}$ demonstrated that anti- $\alpha$-gal antibodies, induced by the presence of Escherichia coli 086:B7 in the gut, are cytotoxic to $\alpha$-gal-expressing Plasmodium sporozoites, and thus protect mice from mosquito-transmitted Plasmodium infection. In the same context, an association was observed between anti- $\alpha$ gal IgM levels and protection from P. falciparum infection in Malian children and adults living in an area of intense malaria transmission ${ }^{(11)}$.

The contributions of microbiota to helminthinduced modulation of autoimmune diseases: Multiple recent investigations have highlighted the promise of helminth-based therapies for the treatment of inflammatory bowel disease (IBD) and coeliac disease. Several studies reported amelioration of clinical symptoms of Crohn's disease and ulcerative colitis in patients subjected to oral administration of T. suis ova ${ }^{(12)}$. Some of the immunoregulatory capacity of worms may be directly or indirectly related to alterations in intestinal microbial communities. Experimental infections with $H$. polygyrus bakeri in a mouse model of IBD revealed a significant expansion of the bacterial family Lactobacillaceae in the ileum of infected mice, which correlated with improved disease outcome ${ }^{(13)}$. Moreover, it was found that microbiota may have a role in the therapeutic potential of helminths in the treatment of allergic diseases. Zaiss et al., ${ }^{(14)}$ reported that chronic infection with the murine helminth $H$. polygyrus bakeri reduced airway inflammation in a house dust mite induced model of allergy by alteration of the intestinal habitat, allowing increased short chain fatty acid production. Also, the transfer of the $H$. polygyrus-modified microbiota alone was sufficient to mediate protection against allergic asthma in uninfected recipient mice.

\section{REFERENCES}

1. Bancroft AJ, Hayes KS, Grencis RK. Life on the edge: the balance between macrofauna, microflora and host immunity. Trends Parasitol 2012; 28(3):93-98.

2. Reynolds LA, Smith KA, Filbey KJ, Harcus $\mathrm{Y}$, Hewitson JP, et al. Commensal-pathogen interactions in the intestinal tract: lactobacilli promote infection with, and are promoted by, helminth parasites. Gut microbes 2014; 5: 522532.

3. Araujo A1, Reinhard KJ, Ferreira LF, Gardner SL. Parasites as probes for prehistoric human migrations? Trends Parasitol 2008; 24: 112-115.

4. Lee SC, Tang MS, Lim YA, Choy SH, Kurtz ZD, Cox $\mathrm{LM}$, et al. Helminth colonization is associated with increased diversity of the gut microbiota. PLoS Negl Trop Dis 2014; 8(5): e2880.

5. Ivanov II, Atarashi K, Manel N, Brodie EL, Shima T, Karaoz U, et al. Induction of intestinal Th17 cells by segmented filamentous bacteria. Cell 2009; 139: 485-498.

6. Atarashi $\mathrm{K}$, Tanoue $\mathrm{T}$, Shima $\mathrm{T}$, Imaoka $\mathrm{A}$, Kuwahara T, Momose Y, et al. Induction of colonic regulatory $\mathrm{T}$ cells by indigenous Clostridium species. Science 2011; 331: 337-341.

7. D'Elia R, Behnke JM, Bradley JE, Else KJ. Regulatory $\mathrm{T}$ cells: a role in the control of helminth-driven intestinal pathology and worm survival. J Immunol 2009; 182:2340-2348.

8. Weinstein PP, Newton WL, Sawyer TK, Sommerville RI. Nematospiroides dubius: development and passage in the germfree mouse, and a comparative study of the free-living stages in germfree feces and conventional cultures. Trans Am Microsc Soc 1969; 88: 95-117.

9. Bär AK, Phukan N, Pinheiro J, Barbosa AS. The interplay of host microbiota and parasitic protozoans at mucosal interfaces: implications for the outcomes of infections and diseases. PLOS Neg Trop Dis 2015; 9(12):e0004176.

10. Yilmaz B, Portugal S, Tran TM, Gozzelino R, Ramos S, Gomes J, et al. Gut microbiota elicits a protective immune response against malaria transmission. Cell 2014;159: 1277-1289. 
11. Yooseph S, Kirkness EF, Tran TM, Harkins DM, Jones MB, Torralba MG, et al. Stool microbiota composition is associated with the prospective risk of Plasmodium falciparum infection. BMC Genomics 2015;16(1): 631.

12. Look P, Lim YAL. Helminths and the microbiota: parts of the hygiene hypothesis. Parasite Immunol 2015 ; 37(6): 314-323.

13. Walk ST. Alteration of the murine gut microbiota during infection with the parasitic helminth
Heligmosomoides polygyrus. Inflamm Bowel Dis 2010;16: 1841-1849.

14. Zaiss MM, Rapin A, Lebon L, Dubey LK, Mosconi $\mathrm{I}$, Sarter $\mathrm{K}$, et al. The intestinal microbiota contributes to the ability of helminths to modulate allergic inflammation. Immunity 2015 ; 43: 998-1010. 